

\title{
Bounded negativity of self-intersection numbers of Shimura curves in Shimura surfaces
}

\author{
Martin Möller and Domingo Toledo
}

\begin{abstract}
Shimura curves on Shimura surfaces have been a candidate for counterexamples to the bounded negativity conjecture. We prove that they do not serve this purpose: there are only finitely many whose self-intersection number lies below a given bound.

Previously (Duke Math. J. 162:10 (2013), 1877-1894), this result was shown for compact Hilbert modular surfaces using the Bogomolov-Miyaoka-Yau inequality. Our approach uses equidistribution and works uniformly for all Shimura surfaces.
\end{abstract}

\section{Introduction}

Let $X$ be a Shimura surface not isogenous to a product, i.e., an algebraic surface which is the quotient of a two-dimensional hermitian symmetric space $G / K$ by an irreducible arithmetic lattice in $G$. The aim of this note is to show that Shimura curves on such a Shimura surface do not provide a counterexample to the bounded negativity conjecture. More precisely we show:

Theorem 0.1. For any Shimura surface $X$ not isogenous to a product and for any real number $M$, there are only finitely many compact Shimura curves $C$ on $X$ with $C^{2}<M$.

The bounded negativity conjecture claims that for any smooth projective algebraic surface $X$ there is a positive constant $B$ so that for any irreducible curve $C$ on $X$ the self-intersection $C^{2}$ is at least $-B$. We emphasize that the above theorem does not decide the validity on any Shimura surface, as there could exist non-Shimura curves with arbitrarily negative self-intersection.

Möller is partially supported by the ERC-StG 257137. Toledo is partially supported by Simons Foundation Collaboration Grant 208853.

MSC2010: primary 14G35; secondary 14J25, 22E40, 37A99, 53C55.

Keywords: bounded negativity, Shimura curves, self-intersections, equidistribution of Shimura curves. 
There are two possibilities for the uniformization of $X$. The first case is Shimura surfaces uniformized by $\mathbb{W}^{2}$. In this case, $G=\mathrm{SL}_{2}(\mathbb{R})^{2}$ and the surfaces are called quaternionic Shimura surfaces if $\Gamma$ is cocompact and Hilbert modular surfaces if $\Gamma$ has cusps. The second case are Shimura surfaces uniformized by the complex 2-ball $\mathbb{B}^{2}$. In this case, $G=\mathrm{SU}(2,1)$ and the surfaces are called Picard modular surfaces. There are compact and noncompact Picard modular surfaces. The assumption on the Shimura surface is necessary, since the theorem is certainly false in the product situation, e.g., for $X=X(d) \times X(d)$ a product of modular curves or a finite quotient of such a surface: the fiber classes give infinitely many curves with self-intersection zero.

While only the case of compact $X$ is relevant to the bounded negativity conjecture, the proofs for noncompact $X$ are the same. When both $X$ and the curves $C$ are allowed to have cusps the proper formulation is needed; see Theorem 3.6.

Theorem 0.1 was proven for compact Shimura surfaces uniformized by $\mathbb{W}^{2}$ in [Bauer et al. 2013]. The methods there, based on the logarithmic BogomolovMiyaoka-Yau inequality, do not extend to the ball quotient case. Here we give a uniform treatment of both cases based on equidistribution results. As in that paper, we obtain as a consequence:

Corollary 0.2. There are only finitely many Shimura curves on $X$ that are smooth.

Intersection numbers of Shimura curves are known to appear as coefficients of modular forms, and coefficients of modular forms are known to grow. This, however, does not directly give a method to prove Theorem 0.1 , since in these modularity statements [Hirzebruch and Zagier 1976; Kudla 1978] the Shimura curves are packaged to reducible curves $T_{N}$ with an unbounded number of components as $N \rightarrow \infty$, while the statement here is for every individual Shimura curve.

\section{Shimura curves on Shimura surfaces not isogenous to a product}

A Shimura surface not isogenous to a product is a connected algebraic surface that can be written as a quotient $X=\Gamma \backslash G / K$, where $G=G_{\mathbb{Q}}(\mathbb{R})$ is the set of $\mathbb{R}$-valued points in a connected semisimple $\mathbb{Q}$-algebraic group $G_{\mathbb{Q}}, K \subset G$ is a maximal compact subgroup and $\Gamma$ is an irreducible arithmetic lattice in $G$. Here a lattice is called irreducible if it does not have a finite-index subgroup that splits as a product of two lattices.

Our geometric definition of Shimura varieties differs from the arithmetic literature on this subject, where Shimura varieties are typically not connected. It is the point of view of the bounded negativity conjecture that requires one to deal with irreducible components of the objects in question. Note that we do not require $\Gamma$ to be a congruence subgroup either. 
Definition. Let $H_{\mathbb{Q}}$ be a $\mathbb{Q}$-algebraic group, $\Delta$ an arithmetic lattice in $H_{\mathbb{Q}}$, and $\tau: H_{\mathbb{Q}} \rightarrow G_{\mathbb{Q}}$ a $\mathbb{Q}$-morphism such that $\tau(\Delta) \subset \Gamma$. Suppose the $\tau$-preimage of a maximal compact subgroup $K \subset G_{\mathbb{R}}$ is a maximal compact subgroup $K_{H} \subset$ $H=H_{\mathbb{Q}}(\mathbb{R})$. Then the algebraic curve $C$ in $X$ given by $C=\Delta \backslash H / K_{H}$ is called a Shimura curve.

The aim of this section is to compile the list of possible constructions of Shimura surfaces that contain infinitely many Shimura curves and the possible pairs $\left(G_{\mathbb{Q}}, H_{\mathbb{Q}}\right)$. This will be used in the equidistribution theorem in the next section. More precisely, we need that all Shimura curves can be generated as the orbit of a fixed subgroup. For this purpose we write $G=G_{0} \times W$ with $W$ compact and $G_{0}$ without compact factors. There is a corresponding decomposition of the compact subgroup $K=K_{0} \times W$, and also for the Shimura curve $H=H_{0} \times W_{H}$ and $K_{H}=K_{H, 0} \times W_{H}$.

It turns out that there are only two possibilities for $G_{0}$, and, for each of them, we can construct all Shimura curves as follows.

Proposition 1.1. For a given Shimura surface $X=\Gamma \backslash G_{0} / K_{0}=\Gamma \backslash G / K$ not isogenous to a product, there exists a subgroup $H_{0} \cong \mathrm{SL}_{2}(\mathbb{R})$ of $G_{0}$ such that all Shimura curves arise as $C=\Gamma \backslash \Gamma g H_{0} / K_{H_{0}}$ for some $g \in G_{0}$.

We start with the possibilities for $G_{0}$. There are only two hermitian symmetric domains of dimension two. This leads to the following two cases, as in the introduction. In each case we give a description of the possible Shimura surfaces. Here, and elsewhere, the description of the algebraic groups in question will always be given only up to central isogeny.

Case One: $G_{0}=\mathrm{SL}_{2}(\mathbb{R})^{2}$. There two possibilities. Either $G$ is the set of $\mathbb{R}$-points of the $\mathbb{Q}$-algebraic group $G_{\mathbb{Q}}=\operatorname{Res}_{F / \mathbb{Q}}\left(\mathrm{SL}_{2}(A)\right)$ for a quaternion algebra $A$ over a totally real field $F$ which is unramified at exactly two infinite places of $F$, or $G$ is the product $\operatorname{Res}_{F / \mathbb{Q}}\left(\mathrm{SL}_{2}\left(A_{1}\right)\right) \times \operatorname{Res}_{F / \mathbb{Q}}\left(\mathrm{SL}_{2}\left(A_{2}\right)\right)$ for two quaternion algebras $A_{1}, A_{2}$, each unramified at exactly at one infinite place. For the proofs, first remark that these give $F$-forms of $\mathrm{SL}_{2}(\mathbb{R})^{2}$; see, e.g., [Vignéras 1980, IV.1]. That these are the only possibilities follows from the classification of algebraic groups [Tits 1966]. In more detail, the procedure of [Tits 1966, §3.1] reduces the problem to the classification of $F$-forms of $\mathrm{SL}_{2}$. The description in [Serre 1994, III.1.4] of the $F$-forms of $\mathrm{SL}_{2}$ in bijective correspondence with quaternion algebras over $F$ gives the above description of the algebraic groups. In both cases, the maximal compact subgroup $K$ in $G$ is $\mathrm{SO}_{2}(\mathbb{R})^{2}$ times the compact factors of $G_{\mathbb{R}}$.

In the product case, all lattices are reducible, so we can discard this case in view of our irreducibility hypothesis on $X$. In the remaining case, in order obtain an arithmetic lattice $\Gamma \subset G$ one has to fix an order $\mathfrak{O} \subset A$ and let $\mathfrak{O}^{1} \subset \mathfrak{O}$ be the 
elements of reduced norm 1. Then $\Gamma$ is the image in $G$ of a group commensurable with $\mathfrak{O}^{1}$. See, e.g., [Vignéras 1980] for more details.

Case Two: $G_{0}=\mathrm{SU}(2,1)$. In this case the underlying $\mathbb{Q}$-algebraic group is $G_{\mathbb{Q}}=$ $\operatorname{Res}_{F_{0} / \mathbb{Q}}\left(G_{F_{0}}\right)$ ), and from the classification of algebraic groups (over number fields) [Tits 1966; Platonov and Rapinchuk 1994], we see that, in the notation of [Tits 1966, p. 55] $G_{F_{0}}$ must be of type ${ }^{2} A_{2, r}^{(d)}$, where $d \mid 3, d \geq 1$ and $2 r d \leq 3$. In other words, $G_{F_{0}}=\mathrm{SU}(h)$, where $h$ is a hermitian form constructed as follows. Start with a totally real field $F_{0}$ and take a totally complex quadratic extension $F / F_{0}$, i.e., $F$ is a CM field. Then take a central simple division algebra $D$ of degree $d$ (hence dimension $d^{2}$ ) over $F$, with center $F$ and involution $\sigma$ of the second kind (not the identity on $F$ ), and a hermitian form $h$ on $D^{3 / d}$ so that $h$ is isotropic at one real place of $F_{0}$ and definite at all other real places (equivalently, isotropic at one conjugate pair of complex places of $F$, definite at all other pairs).

Thus there are two "types" corresponding to the two possibilities $d=1$ or $d=3$ :

The first type corresponds to $d=1$. Then $D=F$ and $h$ is a hermitian form on $F^{3}$ that is definite except for one pair of places of $F$, interchanged by complex conjugation. Then $\mathrm{SU}(h)$ is indeed a $F_{0}$-algebraic group and the set of $\mathbb{R}$-valued points of $\operatorname{Res}_{F_{0} / \mathbb{Q}}(\mathrm{SU}(h))$ equals $G_{0}$ up to compact factors. The compact subgroup $K$ in $G$ is $S(U(2) \times U(1))$ times the compact factors of $G_{\mathbb{R}}$. Arithmetic lattices $\Gamma$ of the first type are obtained by fixing an order $\mathfrak{O} \subset F$ and taking $\Gamma$ commensurable with $G \cap \mathrm{SL}_{3}(\mathfrak{O})$. The integer $r$ above satisfying $2 r d \leq 3$ is the $F_{0}$-rank of $G_{F_{0}}$, or the dimension of the maximal isotropic subspace of $h$ in $F^{3}$. The lattice is cocompact if and only if $r=0$, and $r=1$ forces $F_{0}$ to be $\mathbb{Q}$.

The second type corresponds to $d=3$; in this case, $D$ is central simple division algebra of degree 3 (dimension 9) over $F$ with an involution "of the second kind". The lattices $\Gamma$ are obtained by fixing an order $\mathfrak{O} \subset D$ and taking $\Gamma$ commensurable with $G \cap \operatorname{SL}(D)$. Observe that in this case the inequality $2 r d \leq 3$ forces $r$ to be 0 , and therefore $\Gamma$ is always cocompact. We will see that lattices of the second type do not have any Shimura curves, so we will not need to consider them.

Shimura curves in $X$ for $\boldsymbol{G}_{\mathbf{0}}=\mathbf{S L}_{2}(\mathbb{R})^{2}$. The Shimura curves in $X$ are totally geodesic complex curves in $X$, so they are projections to $X$ of totally geodesic holomorphic disks $\mathbb{H} \subset \mathbb{W}^{2}$, which in turn are orbits of embeddings of $\mathrm{SL}_{2}(\mathbb{R}) \subset$ $\mathrm{SL}_{2}(\mathbb{R})^{2}$. It is well known that, up to biholomorphic isometries, there are only two classes of such disks: factors and diagonals. By the irreducibility hypothesis, the inclusion into one factor does not come from a morphism of the underlying $\mathbb{Q}$-algebraic groups. So $H_{0} \subset G_{0}$ has to be the diagonal embedding, proving Proposition 1.1 in this case. In fact, the possible embeddings are discussed in great detail in [van der Geer 1988] for Hilbert modular surfaces and in [Granath 2002] for quaternionic Shimura surfaces. 
Shimura curves in $X$ for $\boldsymbol{G}_{\mathbf{0}}=\mathbf{S U}(2,1)$. Fix a Shimura surface $X$ obtained by choosing $F_{0}, F, d, D, \sigma, h, \mathfrak{O} \subset D, \Gamma$. The Shimura curves, being totally geodesic complex curves, are projections to $X$ of orbits in the universal cover of subgroups $H \subset G_{0}$, all isomorphic to $\mathrm{SU}(1,1)$ and standardly embedded in $\mathrm{SU}(2,1)$. The image in $X$ of an $H$-orbit is a Shimura curve if and only if $H \cap \Gamma$ is a lattice in $H$. This happens if and only if $H$ is defined over $F_{0}$, meaning that the underlying algebraic group $G_{F_{0}}$ contains an $F_{0}$-subgroup $H_{F_{0}}$ so that, if $\iota: F_{0} \rightarrow \mathbb{R}$ is the embedding of $F_{0}$ with group of real points $G_{F_{0}, l}(\mathbb{R})$ isomorphic to $G_{0}$, the inclusion $H_{F_{0}, l}(\mathbb{R}) \subset G_{F_{0}, l}(\mathbb{R})$ agrees with $H \subset G_{0}$. There are two cases:

No Shimura curves in Shimura surfaces of the second type. The group $\mathrm{SU}(h)$, for $h$ a hermitian form on a central simple division algebra $D$ over $F$ of degree 3 as above, has no subgroup $H_{F_{0}}$ defined over $F_{0}$ with $H_{F_{0}}(\mathbb{R})=\mathrm{SU}(1,1)$ standardly embedded in $\mathrm{SU}(h)(\mathbb{R})=\mathrm{SU}(2,1)$.

This is well-known to experts, but we do not know a reference (but see [Garibaldi and Gille 2009, Corollary 4.2] for a more general result). Matthew Stover kindly communicated the following proof:

Let $F_{0}, F, D, \sigma$ be as above. The $D$-valued hermitian form $h$ can be taken to be $h(x, y)=\sigma(x) y$, and the group of $F_{0}$-points of the $F_{0}$-group in question is

$$
\mathrm{SU}(D, \sigma)\left(F_{0}\right)=\{x \in D: \sigma(x) x=e, N r d(x)=1\} \subset D,
$$

which gives us an $\mathrm{SU}(2,1)$ as follows: choose an embedding $F \rightarrow \mathbb{C}$, use it to form $D \otimes_{F} \mathbb{C}$, which becomes isomorphic to the algebra $M(3, \mathbb{C})$ of $3 \times 3$ complex matrices, under an isomorphism (unique up to conjugation by SkolemNoether) which takes $\sigma$ to its conjugate-transpose with respect to a hermitian form $h^{\prime}$. Whenever all choices can be made so that $h^{\prime}$ has signature $(2,1)$, the group of real points of $\mathrm{SU}(D, \sigma)$ becomes the standard $\mathrm{SU}(2,1)$. The signature of the hermitian form $h^{\prime}$ depends only on $D, \sigma$ and the embedding $F \rightarrow \mathbb{C}$.

Note that the $F$-algebra $D$ is embedded in the algebra $M(3, \mathbb{C})$ by $x \mapsto x \otimes 1$. The $F$-vector subspace of $M(3, \mathbb{C})$ generated by the subset $\mathrm{SU}(D, \sigma)\left(F_{0}\right)$ is easily seen to be a $\sigma$-stable subalgebra of $M(3, \mathbb{C})$ contained in the division algebra $D$, hence it is itself a division algebra, and easily seen to equal $D$. Suppose $H_{F_{0}}$ is an $F_{0}$-subgroup of $\mathrm{SU}(D, \sigma)$, so that the corresponding inclusion of real points is a standard embedding of $\mathrm{SU}(1,1)$ in $\mathrm{SU}(2,1)$, all inside $M(3, \mathbb{C})$, and let $V$ be the $F$-vector subspace of $M(3, \mathbb{C})$ generated by the $F_{0}$-points of $H_{F_{0}}$. This is a noncommutative division subalgebra of $D$, and it must be a proper subalgebra because $V \otimes_{F} \mathbb{C}$ is a proper subspace of $D \otimes_{F} \mathbb{C}=M(3, \mathbb{C})$. Since $D$ has degree 3 , it has no proper noncommutative $F$-subalgebras, so such subgroups cannot exist. 
Classification of Shimura curves in Shimura surfaces of the first type. In this case, there are always infinitely many Shimura curves. We continue the same notation: choose an embedding of $F$ in $\mathbb{C}$ so that the hermitian form $h$ is isotropic, then extend $h$ from $F^{3}$ to $\mathbb{C}^{3}$. Interpret the unit ball $G_{0} / K_{0} \cong \mathbb{B}^{2} \subset \mathbb{P}^{2}$ as the collection of $h$-negative lines in $\mathbb{C}^{3}$. The Shimura curves in $X$ arise as the quotient of totally geodesic disks $\mathbb{B}^{1} \subset \mathbb{B}^{2}$, and such disks are in bijective correspondence with the $h$-positive lines. Namely, an $h$-positive line $l$ determines the hermitian space $\left(\ell^{\perp},\left.h\right|_{l^{\perp}}\right)$ of signature $(1,1)$ and the corresponding space of negative lines $\mathbb{B}_{l}^{1} \subset \mathbb{B}^{2}$. All geodesic disks arise this way. The groups $G_{\ell}$, the stabilizer of $\ell$ (isomorphic to $U(1,1)$ ) and the subgroup $H_{l}$ fixing $l$ pointwise (isomorphic to $\mathrm{SU}(1,1))$ act on $\left(\ell^{\perp},\left.h\right|_{l^{\perp}}\right)$ and $\mathbb{B}_{\ell}^{1}$, both actions being transitive on $\mathbb{B}_{l}^{1}$. The disk $\mathbb{B}_{\ell}^{1}$ projects to a Shimura curve in $X$ if and only if $H_{\ell} \cap \Gamma$ a lattice in $H_{\ell}$; in turn:

Lemma 1.2. The group $H_{\ell} \cap \Gamma$ is a lattice in $H_{\ell}$ if and only if $\ell$ is an $F$-rational line, that is, $\ell \cap F^{3} \neq\{0\}$.

Proof. Let $v \in \mathbb{C}^{3}$ be a basis vector for $\ell$, and suppose $\Gamma_{\ell}=H_{\ell} \cap \Gamma$ is a lattice in $H_{\ell}$. Since $\Gamma_{\ell}$ fixes $\ell$ pointwise, $v$ is fixed by all $\gamma \in H_{\ell} \cap \Gamma$. Since $\Gamma_{\ell}$ leaves $\ell^{\perp}$ invariant, the remaining eigenvectors of any $\gamma \in \Gamma_{\ell}$ lie in $\ell^{\perp}$. Since the action of $H_{\ell}$ on $l^{\perp}$ is isomorphic to the standard action of $\operatorname{SU}(1,1)$ on $\mathbb{C}^{2}$ and $\Gamma_{\ell}$ is a lattice in $H_{\ell}, \Gamma_{l}$ contains hyperbolic elements. Fix such an element $\gamma$. Then $\gamma(v)=v$ and the remaining eigenvalues of $\gamma$ are of absolute value $\neq 1$. Therefore 1 is a simple eigenvalue of $\gamma$, and thus the space of solutions of $\gamma(v)=v$ is an $F$-rational line, as asserted.

For the converse, suppose that $\ell$ is a rational line, and let $v \in \mathfrak{O}^{3}$ be a primitive vector which is a basis for $\ell$. Let $M_{0}=\mathfrak{O} v$ and $M_{1}=v^{\perp} \cap \mathfrak{D}^{3}$, and let $M=M_{0} \oplus M_{1}$. Then $M$ is an $\mathfrak{O}$-submodule of finite index in $\mathfrak{O}^{3}$. Consequently, $\Gamma$ is commensurable with $\Gamma^{\prime}=\{\gamma \in \mathrm{SU}(h, \mathfrak{O}): \gamma(M)=M\}$ and $\Gamma \cap H_{l}$ is commensurable with $\Gamma_{v}^{\prime}=\left\{\gamma \in \Gamma^{\prime}: \gamma(v)=v\right\}$, which is a lattice in the group $H_{\ell}=H_{v}=\{g \in G: g(v)=v\}$, a group defined over $F_{0}$, and isomorphic (over $\left.F_{0}\right)$ to $\mathrm{SU}\left(\left.h\right|_{M_{1} \otimes F}\right)$. This group in turn is isomorphic over $\mathbb{R}$ to $\mathrm{SU}(1,1)$. Thus $\Gamma \cap H_{\ell}$ is a lattice in $H_{\ell}$ and we obtain a Shimura curve associated to the $\mathbb{Q}$-group $\operatorname{Res}_{F_{0} / \mathbb{Q}}\left(\mathrm{SU}\left(\left.h\right|_{M_{1} \otimes F}\right)\right)$.

End of proof of Proposition 1.1. Choose an orthogonal basis $v_{1}, v_{2}, v_{3}$ for $\mathfrak{O}^{3}$, where $h\left(v_{i}\right)=a_{i} \bar{a}_{i}>0$ for $i=1,2, h\left(v_{3}\right)=-a_{3} \bar{a}_{3}<0$ and $v_{1} \in \ell$. Let $e_{1}, e_{2}, e_{3}$ be the standard basis for $\mathbb{C}^{3}$, let $H=H_{e_{1}} \subset G$ be the subgroup, isomorphic to $\mathrm{SU}(1,1)$, that fixes $e_{1}$, and let $g \in G$ be the linear transformation that takes $e_{i}$ to $v_{i} / a_{i}$. Then $\mathrm{gHg}^{-1}=H_{\ell}$; therefore $H_{\ell}$ is as asserted in Proposition 1.1

Remark. From Lemma 1.2 we see that the collection of Shimura curves in $X$ is parametrized by the $\Gamma$-equivalence classes of primitive positive vectors in $\mathfrak{O}^{3}$, that is, primitive vectors $v \in \mathfrak{O}^{3}$ with $h(v)>0$. The collection of these equivalence classes is commensurable with $\mathrm{SU}(h, F) \backslash \mathbb{P}\left(F^{3}\right)^{+}$, where $\mathbb{P}\left(F^{3}\right)^{+}$denotes the space of $h$-positive lines in $F^{3}$. The class of $h(v)$ gives a well-defined function 
$h: \mathbb{P}\left(F^{3}\right) \rightarrow F_{0}^{*} / N_{F / F_{0}}\left(F^{*}\right)$, the norm residue group. It can be checked that the class of $h(v)$ is a commensurability invariant and that it takes on infinitely many values; hence we get an infinite number of commensurability classes of subgroups of SU $(1,1)$. Observe that the matrix of the conjugating element $g$ of Lemma 1.2 has entries in the finite field extension $F\left(a_{1}, a_{2}, a_{3}\right)$ of $F$.

The compact factors of $G$, necessary for the $\mathbb{Q}$-structure in the definition of a Shimura surface, play no role in the sequel. We thus simplify notation and write $G$ for $G_{0}$ and $H$ for $H_{0}$ from now on.

Elliptic elements and cusps. The bounded negativity conjecture (BNC) originally is a question for smooth compact (projective) surfaces. If $\Gamma$ is cocompact and torsion-free, Shimura surfaces as defined above fall into the scope of this conjecture and the results in the introduction need no explanation.

Any arithmetic lattice contains a neat normal subgroup of finite index. Such subgroups are in particular torsion-free. As quotients by a finite group, the Shimura surfaces come with a ( $\mathbb{Q}$-valued) intersection theory. The BNC can be extended to such surfaces, and Theorem 0.1 needs no further explanation.

If $\Gamma$ is cofinite but not cocompact, our proof of Theorem 0.1 gives a statement about the self-intersection number of the cohomology class of the Shimura curve projected to the complement of the cusp resolution cycles, as we will now explain.

We may suppose that $\Gamma$ is a neat subgroup. Let $X^{\mathrm{BB}}$ be the minimal (Baily-Borel) compactification of $X=\Gamma \backslash G / K$. Since $X$ is not isogenous to a product, $X^{\mathrm{BB}} \backslash X$ has codimension two, and hence $H_{c}^{2}(X, \mathbb{Q}) \cong H^{2}\left(X^{\mathrm{BB}}, \mathbb{Q}\right)$. Let $\pi: Y \rightarrow X^{\mathrm{BB}}$ be a (minimal) smooth resolution of the singularities at the cusps and $j: X \rightarrow Y$ the inclusion. We claim that

$$
H^{2}(Y, \mathbb{Q})=\pi^{*} H^{2}\left(X^{\mathrm{BB}}, \mathbb{Q}\right) \oplus B,
$$

where $B$ is the subspace spanned by cusp resolution curves. Moreover, the direct sum is orthogonal and the intersection form on $B$ is negative-definite. This implies that the sum decomposition is compatible with Poincaré duality, and this will make the arguments in Section 3 work in the noncompact case, too; see Theorem 3.6.

Our claims are stated for the Hilbert modular case in [van der Geer 1988, Sections II.3, VI.1]. In the case of a ball quotient, a neighborhood $W$ of the cusps in $Y$ is a disjoint union of disc bundles over tori, each sitting inside a line bundle of negative degree. It suffices to show that

$$
H_{2}(Y, \mathbb{Q})=H_{2}(W, \mathbb{Q}) \oplus \operatorname{Im}\left(j_{*}: H_{2}(X, \mathbb{Q}) \rightarrow H_{2}(Y, \mathbb{Q})\right)
$$

and then apply duality. By Mayer-Vietoris, it suffices to show that $H_{1}(W \cap X, \mathbb{Q}) \rightarrow$ $H_{1}(W, \mathbb{Q}) \oplus H_{1}(X, \mathbb{Q})$ is injective. This holds true, since the inclusion of a circle bundle into the corresponding disc bundle induces an injection the level of $H_{1}(\cdot, \mathbb{Q})$. 
We remark that the BNC (and intersection numbers in general) are very sensitive to blowups. We leave it to the reader to investigate if Theorem 0.1 also holds on $Y$.

Volume normalization. The hermitian symmetric space $G / K$ comes with a Kähler $(1,1)$-form $\omega$ that we normalize, say, so that the minimum value of the curvature of the associated Riemannian metric is -1 . We continue assuming that $\Gamma$ is a neat subgroup, so that $X$ is a manifold with universal cover $\widetilde{X}=G / K$. Then $\omega \wedge \omega$ provides volume forms on $\widetilde{X}$ and $X$. We let $\operatorname{vol}(X)$ be the volume of the Shimura surface. Rescaling by the volume, we obtain a probability measure $v_{X}$ on $X$ induced from the volume form.

Shimura curves are totally geodesic subvarieties in $X$. Consequently, the restriction of $\omega$ is a Kähler form $\omega_{C}$ on $C$. We let $\operatorname{vol}(C)=\int_{C} \omega_{C}$ be the corresponding volume and $v_{C}$ the probability measure defined by $\omega_{C}$.

We need to extend this to the quotients by smaller compact subgroups. Let $K^{\prime} \subset G$ be a compact subgroup and $K_{H}^{\prime}=K^{\prime} \cap H$. Let $v_{G}$ be the Haar measure on $G$ normalized so that the pushforward to $G / K$ gives the above volume form on $\tilde{X}$ and such that the fibers have volume 1 . From $v_{G}$, we obtain measures $v_{G / K^{\prime}}$ on $G / K^{\prime}$ and finite measures $v_{\Gamma \backslash G / K^{\prime}}$ on $X_{K^{\prime}}=\Gamma \backslash G / K^{\prime}$ with $\operatorname{vol}(X)=\operatorname{vol}\left(X_{K^{\prime}}\right)$.

Similarly we fix a normalization of a Haar measure $v_{H}$ on $H$ by requiring that the fibers of $H \rightarrow H / K_{H}$ have volume 1 and that the pushforward to $H / K_{H}$ is the volume form coming from the metric with curvature -1 , as above.

In this way, given a Shimura curve $C=\Gamma \backslash \Gamma g H / K_{H}$, the pushforward of $v_{H}$ defines a finite measure $v_{C, K^{\prime}}$ on the locally symmetric subspaces $C_{K^{\prime}}=\Gamma \backslash \Gamma g H / K_{H}^{\prime}$ inside $X_{K^{\prime}}$ with $\operatorname{vol}\left(C_{K^{\prime}}\right)=\operatorname{vol}(C)$.

\section{Equidistribution}

There are many sources in the literature that deduce equidistribution for Shimura curves from a Ratner-type theorem (notably [Clozel and Ullmo 2005; Ullmo 2007]). We need a slightly stronger equidistribution result, on $\Gamma \backslash G$ or on $\Gamma \backslash G / K^{\prime}$ for some (not necessarily maximal) compact subgroup $K^{\prime}$ of $G$ rather than on the algebraic surface $X$. This follows along known lines from Ratner's result, or rather the version in [Eskin et al. 1996]. We give a proof avoiding technicalities on Shimura data and focusing on the surface case.

The references above contain as special case the following equidistribution:

Proposition 2.1. Suppose that $X$ is a Shimura surface. If $\left(C_{n}\right)_{n \in \mathbb{N}}$ is a sequence of pairwise different Shimura curves, then $v_{C_{n}} \rightarrow v_{X}$ weakly as $n \rightarrow \infty$.

This is a special case of the following stronger result: 
Proposition 2.2. Suppose that $X=\Gamma \backslash G / K$ is a Shimura surface. Let $K^{\prime} \subset K$ be a closed subgroup, and let $g_{n} \in G$ be a sequence of points so that the orbits $g_{n} H \subset G$ project to pairwise-distinct Shimura curves $C_{n}$ in $X$. Then on $X^{\prime}=\Gamma \backslash G / K^{\prime}$ the sequence of probability measures $v_{C_{n}, K^{\prime}}$ converges weakly to $v_{\Gamma \backslash G / K^{\prime}}$ as $n \rightarrow \infty$.

Corollary 2.3. Suppose that $X=\Gamma \backslash G / K$ is a Shimura surface. If $\left(C_{n}\right)_{n \in \mathbb{N}}$ is a sequence of pairwise different Shimura curves, then $\operatorname{vol}\left(C_{n}\right) \rightarrow \infty$ as $n \rightarrow \infty$.

Proof of Corollary 2.3. With the above volume normalization, it suffices to prove the claim for the lifts of the Shimura curves $C_{n}$ to $X^{\prime \prime}=\Gamma \backslash G$. Let $C_{n}^{\prime \prime}$ denote these lifts. We apply the preceding proposition for $K^{\prime}=\{e\}$. Equidistribution implies in particular that Shimura curves are dense; i.e., for any finite collection of open sets $U_{i}, i \in I$, there exists $N_{0}$ such that for $n>N_{0}$ the intersection $C_{n}^{\prime \prime} \cap U_{i}$ is nonempty for all $i$. Since $X^{\prime \prime}$ is foliated by $H$-orbits and $v_{G}$ is locally the product of $v_{H}$ and a transversal measure, it suffices to take for $U_{i}$ sufficiently many open sets locally trivializing the foliation, namely $U_{i}=V_{i} \times W_{i}$ with $V_{i}$ an $H$-orbit, such that $v_{H}\left(V_{i}\right)=$ $O(1)$ but the transversal measure of $W_{i}$ is $O\left(1 / n^{2}\right)$. Then we can fit $O(n)$ such sets into $X$, and each time $C_{n}^{\prime \prime}$ intersects some $U_{i}$ it picks up a volume of $O(1)$.

Proof of Proposition 2.2. We first observe that, if the proposition holds for $K^{\prime}=\{e\}$, then it holds for any other $K^{\prime} \subset K$. Namely, under the projection $\pi: X^{\prime \prime}=\Gamma \backslash G \rightarrow$ $X^{\prime}=\Gamma \backslash G / K^{\prime}$, we have, by the volume normalization above, that the pushforward measures satisfy $\pi_{*}\left(v_{X^{\prime \prime}}\right)=v_{X^{\prime}}$ and $\pi_{*}\left(v_{C_{n}, e}\right)=v_{C_{n}, K^{\prime}}$. Thus we will assume $K^{\prime}=\{e\}$. For this choice of $K^{\prime}$ we have that $X^{\prime}=\Gamma \backslash G$. Thus we'll simply write $X^{\prime}$ for $\Gamma \backslash G$ and $v_{n}^{\prime}$ for $v_{C_{n}, e}$.

The proof consists of two parts: (1) prove that $v_{n}^{\prime}$ has convergent subsequences $v_{n_{j}}^{\prime}$; (2) prove that the limit of any convergent subsequence must be $v_{X^{\prime}}$.

If $\Gamma$ is cocompact, that is, $X^{\prime}$ is compact, then the space of probability measures on $X^{\prime}$ is compact in the weak-* topology, so $v_{n}^{\prime}$ has a convergent subsequence. If $X$ is not compact, then a subsequence converges to a measure on the one-point compactification $X^{\prime} \cup\{\infty\}$, but these measures may "escape to infinity", e.g., converge to the delta function at $\infty$. An example of this "escape of mass" is given in the introduction to [Eskin et al. 1997]. The main result there is that there is no escape of mass when the image of $Z(H)$ in $X^{\prime}$ is compact (where $Z(H)$ is the centralizer of $H$ in $G$ ). More precisely, compactness of the image of $Z(H)$ in $X^{\prime}$ implies (see [Eskin et al. 1997, Theorem 1.1]) that for every $\varepsilon>0$ there exists a compact subset $W \subset \Gamma \backslash G$ such that every $H$-orbit gives measure at least $1-\varepsilon$ to $W$. Hence the sequence $v_{n}^{\prime}$ indeed converges in the space of probability measures on $X^{\prime}$.

In our situation $Z(H)$ itself is compact: it is finite in Case One and $U(1)$ in Case Two, and thus we always have convergence, thereby proving (1). (Compactness of $Z(H)$ generally holds for Shimura varieties if one discards the obvious exception of product situations; see [Ullmo 2007].) 
To prove (2) we may assume $v_{n}^{\prime}$ converges weakly to a probability measure $v^{\prime}$; we must prove $v^{\prime}=v_{X^{\prime}}$. This follows a pattern which is by now standard: (i) use, as in [Eskin et al. 1996], Ratner's theorem on unipotent flows to prove that $v$ is algebraic, i.e., supported on an $L$-orbit of some connected algebraic group $H \subseteq L \subseteq G$ that intersects $\Gamma$ in a lattice; (ii) prove $L=G$. We formulate (i) as the following lemma:

Lemma 2.4. Suppose $v_{n}^{\prime}$ converges weakly to $v^{\prime}$. Then there exists a closed connected subgroup $L, H \subset L \subset G$, such that $v^{\prime}$ is an L-invariant measure supported on $\Gamma \backslash \Gamma c L$ for some $c \in G$ and such that $c^{-1} \Gamma c \cap L$ is a lattice in $L$. Moreover, there exist a sequence $x_{n} \in \Gamma g_{n} H$ converging to $c$ and an $n_{0}$ such that $c L c^{-1}$ contains the subgroup generated by $x_{n} H x_{n}^{-1}$ for $n \geq n_{0}$.

We formulated this lemma following closely the wording of [Eskin and Oh 2006, Proposition 2.1] (see also [Eskin et al. 1996, Theorem 1.7]) because it can be proved from [Mozes and Shah 1995, Theorem 1.1] in same way. Namely, start from the fact that $v_{n}^{\prime}$ is supported on the $H$-orbit $\Gamma \backslash \Gamma g_{n} H \subset \Gamma \backslash G$, which is isomorphic to $\left(g_{n}^{-1} \Gamma g_{n} \cap H\right) \backslash H$ and is $H$-invariant. Since $g_{n}^{-1} \Gamma g_{n}$ is a lattice in $H$, which, in our case, is locally isomorphic to $\operatorname{SL}(2, \mathbb{R})$, we can choose a unipotent one-parameter subgroup $u(t)$ in $H$ and apply the Moore ergodicity theorem, as in the proof of [Eskin and Oh 2006, Proposition 2.1], to show that $v_{n}^{\prime}$ is an ergodic $u(t)$-invariant measure, thus checking that the first hypothesis of [Mozes and Shah 1995, Theorem 1.1] is satisfied. We continue, in this way, following the proof of [Eskin and Oh 2006, Proposition 2.1] until the proof of Lemma 2.4 is complete.

Finally the groups $x_{n} H x_{n}^{-1}$ cannot all be equal to $H$, since this would give $\gamma_{n} \in \Gamma$ so that $g_{n} H g_{n}^{-1}=\gamma_{n} H \gamma_{n}^{-1}$, contradicting the hypothesis that the curves $C_{n}$ are pairwise different. We conclude that $H \subsetneq L$ and thus $L=G$ by the following lemma.

Lemma 2.5. Let $(G, H)$ be as in Case One or Case Two. If $L$ is a connected real Lie group with $H \subsetneq L \subset G$ and $\Gamma \cap L$ is a lattice in $L$, then $L=G$.

Proof. This is easily verified on the level of Lie algebras. Since Lie $(L)$ contains an element not in $\operatorname{Lie}(H)$, bracketing with suitable elements of $\operatorname{Lie}(H)$ allows one to produce a generating set of $\operatorname{Lie}(G)$.

\section{The current of integration of a Shimura curve}

Any Shimura curve $C$, in fact any codimension-one subvariety of the Shimura surface $X$, defines a closed $(1,1)$-current on $X$. On the other hand, the Shimura surface comes with a natural $(1,1)$-form, the Kähler form $\omega$. The aim of this section is to translate the equidistribution result (a convergence of measures) into a convergence statement for the classes of these currents, suitably normalized. We start with the compact case and explain at the end of this section the necessary modification in the noncompact case. Recall that a $(1,1)$-current on a complex 
surface $X$ is a continuous linear functional on $A_{c}^{1,1}(X)$, the space of compactly supported $(1,1)$-forms on $X$. This space $\left(A_{c}^{1,1}(X)\right)^{\vee}$ contains both the complex curves $C \subset X$ and the smooth forms $\eta \in A^{1,1}(X)$ by the formulas

$$
C \rightarrow\left(\alpha \rightarrow \int_{C} \alpha\right), \quad \eta \rightarrow\left(\alpha \rightarrow \int_{X} \eta \wedge \alpha\right) \quad \text { for all } \alpha \in A_{c}^{1,1}(X) .
$$

The cohomology of $X$ can be computed either from the complex of forms or from the complex of currents. Recall also that, if $X$ is Kähler and $\omega$ denotes the Kähler form, then $\operatorname{vol}(X)=\int_{X} \omega \wedge \omega$, the Kähler form of $C$ is $\omega_{C}=\left.\omega_{X}\right|_{C}$ and $\operatorname{vol}(C)=\int_{C} \omega_{C}$.

Proposition 3.1. Let $X=\Gamma \backslash G / K$ be a smooth Shimura surface and let $g_{n} \in G$ be any sequence of points such that the Shimura curves $C_{n}=\Gamma \backslash \Gamma g_{n} H / K$ are pairwise distinct. Then

$$
C_{n} / \operatorname{vol}\left(C_{n}\right) \rightarrow \omega \quad \text { in } A_{c}^{1,1}(X)^{\vee}, \quad \text { hence in } H^{1,1}(X) .
$$

This and the finite-dimensionality of the Picard group allows us to deduce our main result.

Corollary 3.2. Let $X=\Gamma \backslash G / K$ be a compact, smooth Shimura surface and let $g_{n} \in G$ be any sequence of points such that the Shimura curves $C_{n}=\Gamma \backslash g_{m} H / K$ are pairwise distinct. Then

$$
C_{n}^{2} \sim \operatorname{vol}(X) \operatorname{vol}\left(\Gamma \backslash \Gamma g_{n} H\right)^{2} \quad \text { for } n \rightarrow \infty .
$$

In particular, for any $M$, there are only finitely many Shimura curves $C$ on $X$ with $C^{2}<M$.

Proof. For the first statement, fix a basis $\gamma_{0}=\omega, \gamma_{1}, \ldots, \gamma_{s}$ of $H^{1,1}(X)$. Taking $\gamma_{i}$ for $i \geq 1$ orthogonal to $\gamma_{0}$, we may suppose that the dual basis is $\lambda^{-1} \omega=$ $\gamma_{0}^{\vee}, \gamma_{1}^{\vee}, \ldots, \gamma_{s}^{\vee}$ for some $\lambda \in \mathbb{C}$; in fact, $\lambda=\int_{X} \omega \wedge \omega=\operatorname{vol}(X)$. If $C$ is a curve in $X$, thus representing a $(1,1)$-class, the Poincaré dual is represented by

$$
\operatorname{PD}(C)=\sum_{i=0}^{s}\left(\int_{C} \gamma_{i}\right) \gamma_{i}^{\vee} .
$$

Consequently, letting $A_{n}=\operatorname{vol}\left(C_{n}\right)$, by Proposition 3.1,

$$
\begin{aligned}
& \frac{1}{A_{n}^{2}} C_{n} \cdot C_{n}=\frac{1}{A_{n}^{2}} \int_{C_{n}} \operatorname{PD}\left(C_{n}\right)=\sum_{i=0}^{s}\left(\frac{1}{A_{n}} \int_{C_{n}} \gamma_{i}\right)\left(\frac{1}{A_{n}} \int_{C_{n}} \gamma_{i}^{\vee}\right) \\
& \longrightarrow \sum_{i=0}^{s}\left(\int_{X} \omega \wedge \gamma_{i}\right)\left(\int_{X} \omega \wedge \gamma_{i}^{\vee}\right)=\lambda=\operatorname{vol}(X) .
\end{aligned}
$$

The second statement follows from the first and from Corollary 2.3. 
Integrating on the projectivized tangent bundle. We now prepare for the proof of Proposition 3.1. For this purpose we work on the universal cover $\widetilde{X}=G / K$ of $X$. First of all, for any (two-dimensional) Kähler manifold $X$ there is a natural map

$$
\mathbb{P} T \tilde{X} \rightarrow \Lambda_{1,1} T \tilde{X}=\left(\Lambda^{1,1} T^{*} \tilde{X}\right)^{\vee},
$$

defined pointwise at any $x \in \tilde{X}$ by $[v] \mapsto v \wedge \bar{v} /|v|^{2}$ for $v \in T_{x} \widetilde{X} \backslash\{0\}$. Dually, an element $\alpha \in\left(\Lambda^{1,1} T^{*} \widetilde{X}\right)$ defines a real-valued function

$$
\varphi_{\alpha}: \mathbb{P} T \tilde{X} \rightarrow \mathbb{R}, \quad \varphi_{\alpha}([v])=\alpha\left(\frac{v \wedge \bar{v}}{|v|^{2}}\right)
$$

Using this map we can write the intersection with $\alpha$ as the integral of a realvalued function against the volume form of $\mathbb{P} T X$. In Case Two, $\mathbb{P} T \tilde{X}=G / K^{\prime}$ is a homogeneous space with an invariant volume, where $K^{\prime}=\mathrm{U}(1) \times \mathrm{U}(1)$. In Case One, we will need to pass to a $G$-invariant real subbundle of $\mathbb{P} T \widetilde{X}$, also of the form $G / K^{\prime}$ for $K^{\prime}=\mathrm{U}(1)$.

We start with Case Two. Recall that we scaled the Kähler form $\omega$ so that $\operatorname{vol}(X)=\int_{X} \omega \wedge \omega$.

Lemma 3.3. Let $X$ be a two-dimensional Kähler manifold, choose a two-form $\eta$ on $\mathbb{P} T X$ that restricts to the area form $\eta_{x}$ of each fiber $\mathbb{P} T_{x} X, x \in X$, scaled to give total area 1 to each fiber. Then, for all $(1,1)$-forms $\alpha$ on $X$ and for each $x \in X$, we have

$$
(\omega \wedge \alpha)_{x}=\left(\int_{\mathbb{P} T_{x} X} \varphi_{\alpha} \eta_{x}\right)(\omega \wedge \omega)_{x}
$$

Therefore we have

$$
\int_{X} \omega \wedge \alpha=\int_{\mathbb{P} T X} \varphi_{\alpha} \eta \wedge \omega \wedge \omega
$$

where we have written simply $\omega$ for the pullback to $\mathbb{P} T X$ of the form $\omega$ on $X$.

Proof. In suitable local coordinates at $x$, the Kähler form at $x$ is

$$
\omega_{x}=\frac{\sqrt{-1}}{2}\left(d z_{1} \wedge d \bar{z}_{1}+d z_{2} \wedge d \bar{z}_{2}\right)
$$

Writing $\alpha=\frac{\sqrt{-1}}{2} \sum \alpha_{i} d z_{i} \wedge d \bar{z}_{j}$, we have (suppressing the factors of $\frac{\sqrt{-1}}{2}$ )

$$
(\omega \wedge \alpha)_{x}=\left(\alpha_{1 \overline{1}}+\alpha_{2 \overline{2}}\right)\left(d z_{1} \wedge d \bar{z}_{1} \wedge d z_{2} \wedge d \bar{z}_{2}\right)=\frac{\alpha_{1 \overline{1}}+\alpha_{2 \overline{2}}}{2}(\omega \wedge \omega)_{x} .
$$


On the other hand, if we let $e_{1}, e_{2}$ denote the basis for $T_{x} X$ dual to $d z_{1}, d z_{2}$, and write $v=v_{1} e_{1}+v_{2} e_{2} \in T_{x} X$, the first factor of the right-hand side is

$$
\begin{aligned}
\int_{\mathbb{P} 1} & \alpha\left(\frac{\left(v_{1} e_{1}+v_{2} e_{2}\right) \wedge \overline{\left(v_{1} e_{1}+v_{2} e_{2}\right)}}{\left|v_{1}\right|^{2}+\left|v_{2}\right|^{2}}\right) \eta_{x} \\
& =\alpha_{1 \overline{1}} \int_{\mathbb{P} 1} \frac{\left|v_{1}\right|^{2}}{\left|v_{1}\right|^{2}+\left|v_{2}\right|^{2}} \eta_{x}+\alpha_{2 \overline{2}} \int_{\mathbb{P}^{1}} \frac{\left|v_{2}\right|^{2}}{\left|v_{1}\right|^{2}+\left|v_{2}\right|^{2}} \eta_{x}+\int_{\mathbb{P} 1} \frac{2 \operatorname{Im}\left(\alpha_{1 \overline{2}} v_{1} \bar{v}_{2}\right)}{\left|v_{1}\right|^{2}+\left|v_{2}\right|^{2}} \eta_{x} .
\end{aligned}
$$

The involution $\left(v_{1}: v_{2}\right) \rightarrow\left(v_{2}: v_{1}\right)$ is an isometry of $\mathbb{P}^{1}$. The last integrand is sent to its negative by this involution, so the last integral vanishes. The first two integrals are interchanged by this involution, therefore they are equal. Since the sum of the two integrands is visibly identically 1 , each of the first two integrals has value $\frac{1}{2}$. Thus the first two terms give $\frac{1}{2} \alpha_{1 \overline{1}}$ and $\frac{1}{2} \alpha_{2 \overline{2}}$ respectively, hence the first statement of the lemma follows. The second follows from the first and Fubini's theorem.

Remark. The first statement in the lemma is equivalent to the well-known fact in linear algebra that the trace of a hermitian matrix equals the average value over the unit sphere of the associated hermitian form.

Corollary 3.4. If $X$ is a Shimura surface covered by the ball, then for all $(1,1)$ forms $\alpha$ on $X$ we have

$$
\int_{X} \omega \wedge \alpha=\int_{\mathbb{P} T X} \varphi_{\alpha} d v_{\Gamma \backslash G / K^{\prime}}
$$

where $v_{\Gamma \backslash G / K^{\prime}}$ is the volume form on $\mathbb{P} T X$ introduced above.

Proof. If $\widetilde{X}=\mathbb{B}^{2}=G / K$, then $\eta \wedge \omega \wedge \omega$ in Lemma 3.3 is a $G$-invariant volume form on $\mathbb{P} T \tilde{X}$. Moreover, $\omega$ and $\eta$ have been scaled to give the correct normalization.

Now we address the corresponding statement in Case One. If the Shimura surface $X$ is covered by $\mathbb{U}^{2}$, then $\mathbb{P} T \tilde{X}$ is no longer a homogeneous space for $G$, but it has some natural homogeneous subbundles. Equivalently, the action of $K$ on $\mathbb{P} T_{x} \widetilde{X} \cong \mathbb{P}^{1}$ is not transitive, but has some distinguished orbits: two zerodimensional orbits, corresponding to the tangents to the two factors of $\mathbb{W}^{2}$, and an orbit of real dimension 1 corresponding to the graphs of isometries between the two factors. Explicitly, if we choose coordinates $z_{1}, z_{2}$ as above, this time adapted to the product structure of $\tilde{X}$, and with dual basis $e_{1}, e_{2}$ each tangent to one of the factors, and writing $v=v_{1} e_{1}+v_{2} e_{2}$ as above, the action of $K \cong \mathrm{U}(1) \times \mathrm{U}(1)$ on $\mathbb{P} T_{x} \widetilde{X} \cong \mathbb{P}^{1}$ leaves invariant the points with homogeneous coordinates $(1: 0)$ and $(0: 1)$ and the real submanifold $\left\{\left(v_{1}: v_{2}\right):\left|v_{1}\right|=\left|v_{2}\right|\right\}=\left\{\left(1: e^{i \theta}\right)\right\} \cong S^{1}$.

Let us call this submanifold $\mathbb{S} T_{x} \widetilde{X}$ and let $\mathbb{S} T \widetilde{X} \cong G / K^{\prime}$ denote the corresponding bundle over $\tilde{X} \cong G / K$ with fiber $K / K^{\prime} \cong \mathbb{S} T_{x} \widetilde{X} \cong S^{1}$. Then a calculation just as in the proof of Lemma 3.3 gives us: 
Lemma 3.5. Let $X$ be a Shimura surface covered by $\mathbb{\boxplus}^{2}$, choose a one-form $\eta$ on $\mathbb{S} T X$ that restricts to the angle form $\eta_{x}=d \theta$ of each fiber $\mathbb{S} T_{x} X$, scaled to give total area 1 to each fiber. Then, for any $(1,1)$ form $\alpha$ on $X$ and for each $x \in X$, we have

$$
(\omega \wedge \alpha)_{x}=\left(\int_{\mathbb{S} T_{x} X} \varphi_{\alpha} \eta_{x}\right)(\omega \wedge \omega)_{x}
$$

Therefore we have

$$
\int_{X} \omega \wedge \alpha=\int_{\mathbb{S} T X} \varphi_{\alpha} \eta \wedge \omega \wedge \omega=\int_{\mathbb{S} T X} \varphi_{\alpha} d \nu_{\Gamma \backslash G / K^{\prime}}
$$

where $\nu_{\Gamma \backslash G / K^{\prime}}$ is the volume form on $\$ \mathbb{S} X$ introduced above.

Proof of Proposition 3.1. To show convergence in $H^{1,1}(X)$ it suffices to show that

$$
\frac{1}{\operatorname{vol}\left(C_{n}\right)} \int_{C_{n}} \alpha \rightarrow \int_{X} \omega \wedge \alpha
$$

for any $\alpha \in H^{1,1}(X)$. In Case Two, by Corollary 3.4 it suffices to show that

$$
\frac{1}{\operatorname{vol}\left(C_{n}\right)} \int_{C_{n}} \alpha \rightarrow \int_{\mathbb{P} T X} \varphi_{\alpha} d v_{\Gamma \backslash G / K^{\prime}}
$$

A local verification, just using the definition of $\varphi_{\alpha}$ and the fact that $v_{C_{n}, K^{\prime}}$ was defined to give measure 1 to the fibers $K / K^{\prime}$, implies that $\int_{C_{n}} \alpha=\int_{\mathbb{P} T C_{n}} \varphi_{\alpha} d \nu_{C_{n}, K^{\prime}}$. Since $v_{C_{n}, K^{\prime}}$ is supported on $\mathbb{P} T C_{n} \subset \mathbb{P} T X$, it is thus sufficient to show that

$$
\int_{\mathbb{P} T X} \varphi_{\alpha} d v_{C_{n}, K^{\prime}} \rightarrow \int_{\mathbb{P} T X} \varphi_{\alpha} d v_{\Gamma \backslash G / K^{\prime}}
$$

We have reformulated our claim in terms of a convergence of measures, integrating against a globally defined function $\varphi_{\alpha}$. Proposition 2.2 completes the proof. In Case One, the proof is the same, replacing $\mathbb{P} T X$ by $\mathbb{S} T X$ and the reference to Corollary 3.4 by Lemma 3.5.

The noncompact case. Recall that we denoted by $Y$ a minimal resolution of the singularities of the Baily-Borel compactification $X^{\mathrm{BB}}$. By [Mumford 1977, Theorem 3.1, Proposition 1.1], the Kähler class $\omega$ extends to a closed current on $Y$. Moreover, $\omega \in \pi^{*} H^{2}\left(X^{\mathrm{BB}}, \mathbb{Q}\right)$ by [Mumford 1977, Proposition 3.4(b)]. The statement of Proposition 3.1 now reads

$$
p_{B^{\perp}}\left(C_{n}\right) / \operatorname{vol}\left(C_{n}\right) \rightarrow \omega \text { in } \pi^{*} H^{2}\left(X^{\mathrm{BB}}, \mathbb{Q}\right),
$$

where $p_{B}^{\perp}$ is the orthogonal projection onto the complement of $B$. The same proof as above works. In order to show the analog

$$
\left(p_{B^{\perp}} C_{n}\right)^{2} \sim \operatorname{vol}\left(\Gamma \backslash \Gamma g_{n} H\right)^{2} \text { for } n \rightarrow \infty
$$


of Corollary 3.2, we apply Poincaré duality to $\pi^{*} H^{2}\left(X^{\mathrm{BB}}, \mathbb{Q}\right)$. Since this is a perfect pairing, the proof of Corollary 3.2 applies without changes:

Theorem 3.6. For $X$ as above and for any real number $M$, there are only finitely many Shimura curves $C$ on $X$ with $\left(p_{B^{\perp}} C\right)^{2}<M$.

In particular, for the collection of compact Shimura curves in $X$, we obtain Theorem 0.1 .

\section{Acknowledgements}

This note is the result of the Mathematisches Forschungsinstitut Oberwolfach mini-workshop "the bounded negativity conjecture". We thank the organizers of this workshop and the MFO administration for organizing the simultaneous miniworkshop "Kähler groups", which brought the two authors together. We thank Alex Eskin for discussing details on equidistribution results, and Madhav Nori and Matthew Stover for discussions on algebraic group details. We thank the referee for a thorough report and helpful suggestions. Toledo thanks Misha Gromov for a conversation in 1990 on the idea that Shimura curves approximate the Kähler class.

The paper [Koziarz and Maubon 2014], with some overlap with ours, also resulted from the MFO workshop.

\section{References}

[Bauer et al. 2013] T. Bauer, B. Harbourne, A. L. Knutsen, A. Küronya, S. Müller-Stach, X. Roulleau, and T. Szemberg, "Negative curves on algebraic surfaces", Duke Math. J. 162:10 (2013), 1877-1894. MR 3079262 Zbl 1272.14009

[Clozel and Ullmo 2005] L. Clozel and E. Ullmo, "Équidistribution de sous-variétés spéciales", Ann. of Math. (2) 161:3 (2005), 1571-1588. MR 2006j:11083 Zbl 1099.11031

[Eskin and Oh 2006] A. Eskin and H. Oh, "Ergodic theoretic proof of equidistribution of Hecke points”, Ergodic Theory Dynam. Systems 26:1 (2006), 163-167. MR 2006j:11068 Zbl 1092.11023

[Eskin et al. 1996] A. Eskin, S. Mozes, and N. Shah, "Unipotent flows and counting lattice points on homogeneous varieties”, Ann. of Math. (2) 143:2 (1996), 253-299. MR 97d:22012 Zbl 0852.11054

[Eskin et al. 1997] A. Eskin, S. Mozes, and N. Shah, "Non-divergence of translates of certain algebraic measures", Geom. Funct. Anal. 7:1 (1997), 48-80. MR 98d:22006 Zbl 0872.22009

[Garibaldi and Gille 2009] S. Garibaldi and P. Gille, "Algebraic groups with few subgroups", J. Lond. Math. Soc. (2) 80:2 (2009), 405-430. MR 2010i:20050 Zbl 1183.20053

[van der Geer 1988] G. van der Geer, Hilbert modular surfaces, Ergebnisse der Mathematik und ihrer Grenzgebiete (3) 16, Springer, Berlin, 1988. MR 89c:11073 Zbl 0634.14022

[Granath 2002] H. Granath, On quaternionic Shimura surfaces, Ph.D. thesis, Chalmers Tekniska Hogskola, 2002, Available at http://search.proquest.com/docview/305513982. MR 2715512

[Hirzebruch and Zagier 1976] F. Hirzebruch and D. Zagier, "Intersection numbers of curves on Hilbert modular surfaces and modular forms of Nebentypus”, Invent. Math. 36 (1976), 57-113. MR 56 \#11909 Zbl 0332.14009 
[Koziarz and Maubon 2014] V. Koziarz and J. Maubon, "On the equistribution of totally geodesic submanifolds in compact locally symmetric spaces and application to boundedness results for negative curves and exceptional divisors", preprint, 2014. arXiv 1407.6561v2

[Kudla 1978] S. S. Kudla, "Intersection numbers for quotients of the complex 2-ball and Hilbert modular forms", Invent. Math. 47:2 (1978), 189-208. MR 80a:10044 Zbl 0399.10030

[Mozes and Shah 1995] S. Mozes and N. Shah, "On the space of ergodic invariant measures of unipotent flows”, Ergodic Theory Dynam. Systems 15:1 (1995), 149-159. MR 95k:58096 Zbl 0818.58028

[Mumford 1977] D. Mumford, "Hirzebruch's proportionality theorem in the noncompact case", Invent. Math. 42 (1977), 239-272. MR 81a:32026 Zbl 0365.14012

[Platonov and Rapinchuk 1994] V. Platonov and A. Rapinchuk, Algebraic groups and number theory, Pure and Applied Mathematics 139, Academic Press, Boston, 1994. MR 95b:11039 Zbl 0841.20046

[Serre 1994] J.-P. Serre, Cohomologie galoisienne, 5th ed., Lecture Notes in Mathematics 5, Springer, Berlin, 1994. MR 96b:12010 Zbl 0812.12002

[Tits 1966] J. Tits, "Classification of algebraic semisimple groups", pp. 33-62 in Algebraic Groups and Discontinuous Subgroups (Boulder, CO 1965), Proc. Sympos. Pure Math. 9, Amer. Math. Soc., Providence, R.I., 1966. MR 37 \#309 Zbl 0238.20052

[Ullmo 2007] E. Ullmo, "Equidistribution de sous-variétés spéciales, II", J. Reine Angew. Math. 606 (2007), 193-216. MR 2008g:11103 Zbl 1137.11043

[Vignéras 1980] M.-F. Vignéras, Arithmétique des algèbres de quaternions, Lecture Notes in Mathematics 800, Springer, Berlin, 1980. MR 82i:12016 Zbl 0422.12008

Communicated by Hélène Esnault

Received 2014-08-16 Revised 2015-03-02 Accepted 2015-04-07

moeller@math.uni-frankfurt.de Institut für Mathematik, Goethe-Universität Frankfurt, Robert-Mayer-Strasse 6-8, D-60325 Frankfurt am Main, Germany

toledo@math.utah.edu

Department of Mathematics, University of Utah, 155 S 1400 E, Salt Lake City, UT 84112, United States 


\section{Algebra \& Number Theory}

msp.org/ant

\section{EDITORS}

MANAGING EDITOR

Bjorn Poonen

Massachusetts Institute of Technology

Cambridge, USA

\author{
EDITORIAL BOARD CHAIR \\ David Eisenbud \\ University of California \\ Berkeley, USA
}

\section{BOARD OF EDITORS}

Georgia Benkart

Dave Benson

Richard E. Borcherds

John H. Coates

J-L. Colliot-Thélène

Brian D. Conrad

Hélène Esnault

Hubert Flenner

Edward Frenkel

Andrew Granville

Joseph Gubeladze

Roger Heath-Brown

Craig Huneke

Kiran S. Kedlaya

János Kollár

Yuri Manin

Barry Mazur

Philippe Michel
University of Wisconsin, Madison, USA

University of Aberdeen, Scotland

University of California, Berkeley, USA

University of Cambridge, UK

CNRS, Université Paris-Sud, France

Stanford University, USA

Freie Universität Berlin, Germany

Ruhr-Universität, Germany

University of California, Berkeley, USA

Université de Montréal, Canada

San Francisco State University, USA

Oxford University, UK

University of Virginia, USA

Univ. of California, San Diego, USA

Princeton University, USA

Northwestern University, USA

Harvard University, USA

École Polytechnique Fédérale de Lausanne
Susan Montgomery

Shigefumi Mori

Raman Parimala

Jonathan Pila

Anand Pillay

Victor Reiner

Peter Sarnak

Joseph H. Silverman

Michael Singer

Vasudevan Srinivas

J. Toby Stafford

Ravi Vakil

Michel van den Bergh

Marie-France Vignéras

Kei-Ichi Watanabe

Efim Zelmanov

Shou-Wu Zhang
University of Southern California, USA

RIMS, Kyoto University, Japan

Emory University, USA

University of Oxford, UK

University of Notre Dame, USA

University of Minnesota, USA

Princeton University, USA

Brown University, USA

North Carolina State University, USA

Tata Inst. of Fund. Research, India

University of Michigan, USA

Stanford University, USA

Hasselt University, Belgium

Université Paris VII, France

Nihon University, Japan

University of California, San Diego, USA

Princeton University, USA

\section{PRODUCTION}

production@msp.org

Silvio Levy, Scientific Editor

See inside back cover or msp.org/ant for submission instructions.

The subscription price for 2015 is US $\$ 255 /$ year for the electronic version, and $\$ 440 /$ year ( $+\$ 55$, if shipping outside the US) for print and electronic. Subscriptions, requests for back issues and changes of subscribers address should be sent to MSP.

Algebra \& Number Theory (ISSN 1944-7833 electronic, 1937-0652 printed) at Mathematical Sciences Publishers, 798 Evans Hall \#3840, c/o University of California, Berkeley, CA 94720-3840 is published continuously online. Periodical rate postage paid at Berkeley, CA 94704, and additional mailing offices.

ANT peer review and production are managed by EditFLOW ${ }^{\circledR}$ from MSP.

\section{PUBLISHED BY}

- mathematical sciences publishers

nonprofit scientific publishing

http://msp.org/

(C) 2015 Mathematical Sciences Publishers 


\section{Algebra \& Number Theory}

Volume $9 \quad$ No. $4 \quad 2015$

Motivic Donaldson-Thomas invariants of small crepant resolutions

ANDREW MORRISON and KENTARO NAGAO

Étale homotopy equivalence of rational points on algebraic varieties

AMBRUS PÁL

Fermat's last theorem over some small real quadratic fields

NUNO FREITAS and SAMIR SIKSEK

Bounded negativity of self-intersection numbers of Shimura curves in Shimura surfaces MARTIN MÖLLER and DOMINGo TOLEDO

Singularities of locally acyclic cluster algebras

Angélica Benito, Greg Muller, Jenna Rajchgot and Karen E. Smith

On an analytic version of Lazard's isomorphism

GEORG TAMME

Towards local-global compatibility for Hilbert modular forms of low weight JAMES NEWTON

Horrocks correspondence on arithmetically Cohen-Macaulay varieties

981

Francesco Malaspina and A. Prabhakar RaO

The Elliott-Halberstam conjecture implies the Vinogradov least quadratic nonresidue conjecture

TERENCE TAO 